\title{
STATE OF THE ART DEVELOPMENTS IN HOT FLAT PRODUCT SURFACE INSPECTION*
}

\author{
Laurent Dore/ ${ }^{1}$ \\ Afchine Nasserian ${ }^{2}$ \\ Sébastien Maillard ${ }^{3}$ \\ Jean-Paul Faure ${ }^{4}$
}

\begin{abstract}
Surface quality monitoring is primarily implemented in the last steps of steel manufacturing before shipment to the end user. However, an increasing trend for also addressing this topic in the upstream stages, such as hot rolling plant, is tackled by Primetals Technologies to give steel producers the key to a global optimized yield management, and efficient "lessons learned" processes. After the last developments of SIAS Next Gen platform, including High Resolution and Near Infra-Red, some site results will be exposed on the very specific high constraints lines such as ESP Endless Strip Production and Plate Mills, and highlights the related most interesting aspects for our customers.

Keywords: Surface inspection; Flatness; Hot rolling mill; Endless strip production.
\end{abstract}

1 Product Manager, Primetals Technologies France SAS, Savigneux Cedex, France.

2 Technical Sales Manager Products, Primetals Technologies France SAS, Savigneux Cedex, France.

3 Head of Technology and Innovation, Primetals Technologies France SAS, Savigneux Cedex, France.

4 Proposal Manager, Primetals Technologies France SAS, Savigneux Cedex, France. 


\section{INTRODUCTION}

For the last few years, Primetals Technologies has continued intensive development in the hot rolling field, for which various specific domains have been explored. This article will describe the latest achievements in surface inspection. The results of two systems will be presented - one installed in a conventional hot strip mill and another in an Arvedi Endless Strip Production line (Arvedi ESP). The hot strip mill example has been selected because of the broad range of products that the mill produces (stainless steels, grain-oriented electrical steels, titanium and nickel alloys, and advanced alloys). The Arvedi ESP plant example, was selected based on its unique production process (hot rolling down to less than $0.8 \mathrm{~mm}$ thick), where extreme production constraints and environment present several challenges (more than 10 hours endless production, gauge platform temperature after the finishing stand reaching more than $80^{\circ} \mathrm{C}$ ). Strip surface quality assessments are just the beginning. The latest trend requires a global quality control approach, which will be introduced in this paper.

\section{STRIP SURFACE INSPECTION AT HOT MILLS}

Following the casting process stage, strip hot rolling is the first important stage to master final surface quality from an end-user standpoint. This is the point where process, metallurgy and surface quality, have to be understood and determined for that purpose.

Addressing surface quality control for the most stringent demands is still typically driven by steel grades produced for home appliances and exposed automotive panel applications. Higher strength material (HSLA, multiphase, etc.) are usually not used in exposed panels and are not subject to the highest surface quality requirements. However this material still requires attention from a surface control standpoint.

The necessity to detect detrimental defects at the earliest stage before further processing is more and more vital to minimize global production costs and reduce claims from end-users. This covers surface aspects and dimensional defects such as pores, shells, holes and cracks; with dimensions down to $0.5 \mathrm{~mm}^{2}$ as now specified for hot rolled coils.

Grades are usually connected to specific types of surface defects (i.e. edge cracks for electrical silicon steel, scale defects for low or ultra-low carbon grades, etc.). In order to get the optimum performance out of the surface detection and classification functionalities, it is crucial to understand the link between grades/processes and the most probable defects. First series defects originate from the casting process and cannot be fully mitigated at the hot rolling stage. Second series defects are due to improper rolling conditions.

The latest challenges are related to flatness quality control, especially for recent plant generation (direct casting \& rolling, endless or semi-endless processes) able to produce hot rolled material in dimensional ranges never experienced so far. In hot conditions, steel can be rolled down to a thickness of $0.8 \mathrm{~mm}$ or less as Arvedi ESP type plants are now achieving. This generates new challenges in terms of controlling surface defects and flatness 


\section{EXCEPTIONAL MILLS: SPECIAL METALS HOT STRIP MILL AND ENDLESS STRIP PRODUCTION LINE}

\subsection{Surface Inspection System at the Hot Strip Mill and Installation Description}

The below Surface Inspection Automated System SIAS $尺$ is located in a new HotRolling Mill, with a new six-stand hot strip mill fed from a 125-ton argon-oxygen decarburization (AOD) refining and continuous caster and dedicated to the production of a wide range of metals and alloys.

The top side is composed of one 4096 pixel CCD high sensitivity (10 $\mu$ pixel cell) camera and a $3.2 \mathrm{~m}$ long diffused type Blue LED light source. The unusual length of the light source is due to the significant distance between the latter and the strip.

The bottom side is composed of two 4096 pixel CCD (10 $\mu$ pixel cell) cameras. The same lighting, with a smaller light source length of $2.57 \mathrm{~m}$ is used.

The main Results should be presented in a concise and logically ordered way. Be sparing in the use of tables and ensure that the data presented in them do not duplicate results described elsewhere in the article. Example of Table (Figure 1).

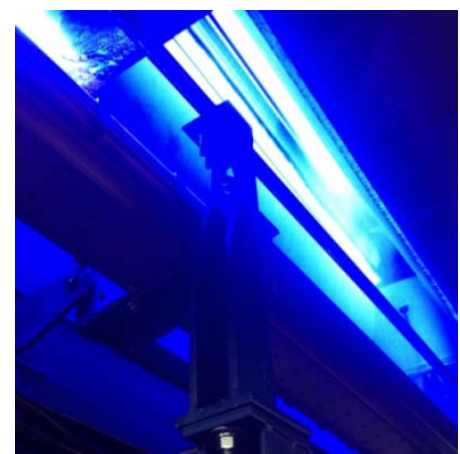

Bottom inspection

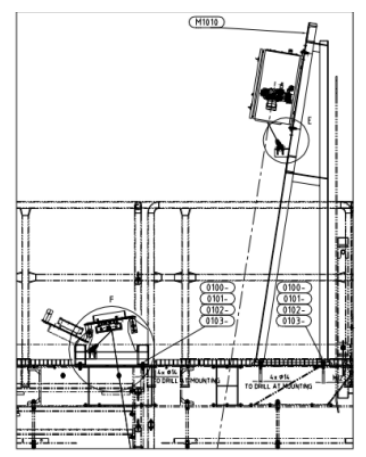

Top inspection

Figure 1. SIAS installation principles

\subsection{Surface Inspection System at the Endless Strip Production line and Installation Description}

Primetals Technologies Arvedi ESP Endless Strip Production line produces thicknesses from $0.8 \mathrm{~mm}$ up to $25.4 \mathrm{~mm}$, with an exit speed of $900 \mathrm{mpm}$. Strip is produced continuously for more than 10 hours, with complete range of steel grades, such as CQ, HSAL, AHSS steels.

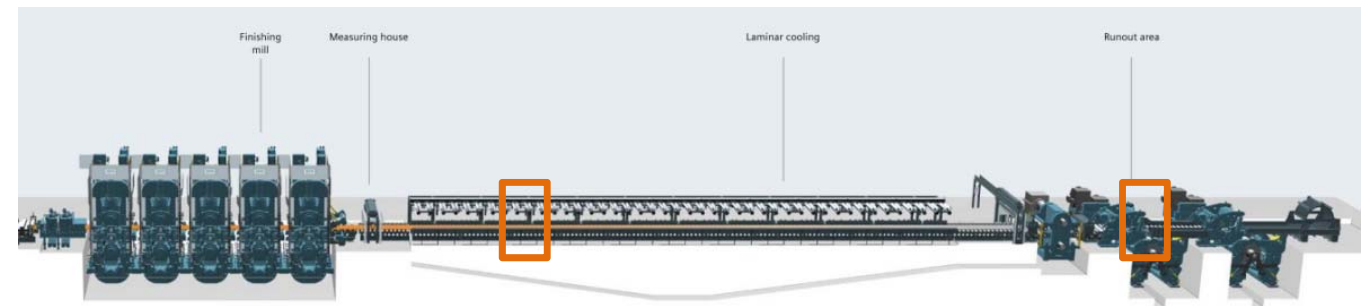

Figure 2. Arvedi Endless Strip Production line, with location of top and bottom surface inspection systems 


\subsection{System, location and performances for Endless Strip Production line}

The latest generation NextGen SIAS system, is composed of one unique server equipped with multiple high-speed FPGA based processing boards. This is the backbone of the implementation of high resolution top \& bottom channels, allowing a pixel resolution of $0.35 \times 0.35 \mathrm{~mm}^{2}$, plus an additional NIR "Near Infra-Red channel" completing the top side.

Lighting is composed of multiple LED panels arranged in a dome shaped configuration, which provides the most uniform lighting and best image quality.

Top side inspection is located after the finishing mill and before the laminar cooling process, where the strip average temperature is not lower than $900^{\circ} \mathrm{C}$ (necessary condition for proper NIR channel working). It is to be noted that in Arvedi ESP lines, the area temperature where the top SIAS is installed (gauge platform) can reach more than $80^{\circ} \mathrm{C}$ for few hours as the hot strip production runs continuously without interruption (in opposition to conventional HSM with discontinued production).

Bottom side inspection is located after the laminar cooling process and before the downcoiler, and the usual environmental difficulties are present (scale/water falling somehow less severe compared to traditional hot strip mills, continuous heating condition here more severe than usual HSM).

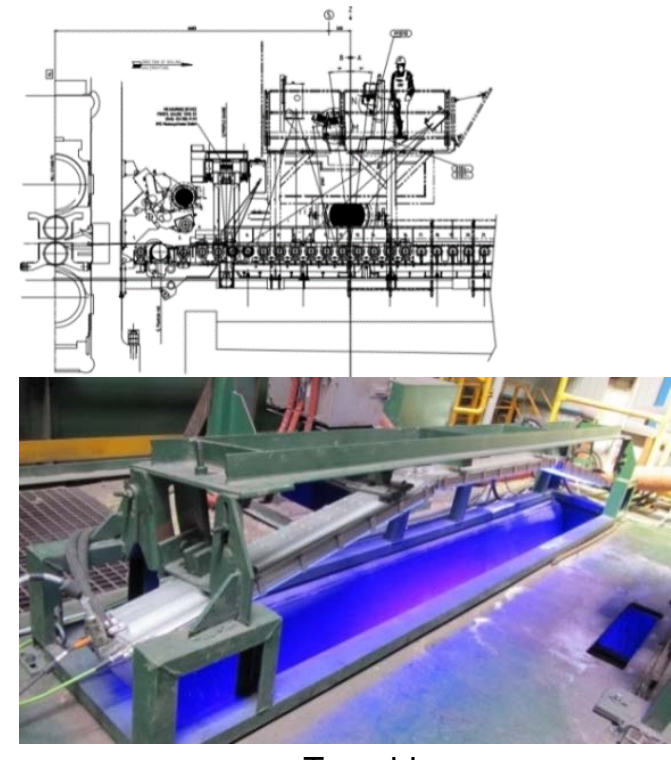

Top side

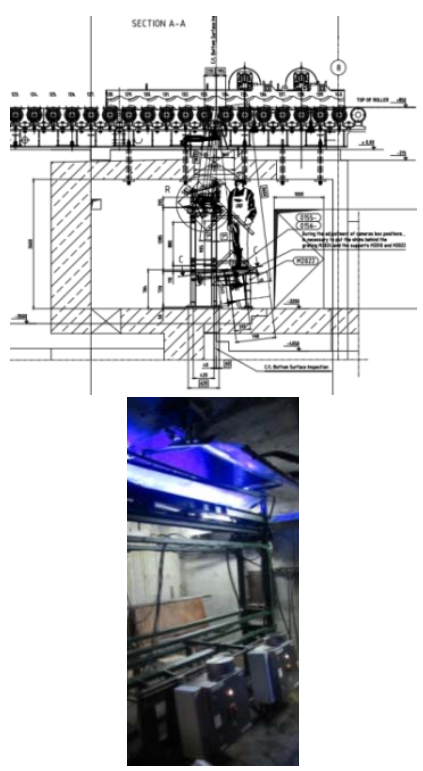

Bottom side

Figure 3. Top side and bottom side of NextGen SIAS system

\section{HOT STRIP DEFECTS MONITORED AND CLASSIFIED}

\subsection{Typical defects monitored and classified}

Product mixes are discretized into several groups, which are determined by parameter sets. This discretization is based on the surface affinity and defect monitoring targets. This typically leads to 5 sets for "simple" cases to 30 for very broad mixes.

Each of the parameter sets rules approximately 30 to 40 different classes which are monitored individually and automatically classified by the inspection system. 
Typical classes of this hot strip mill, as defined by quality engineers, include: splicing, scale, dirt, salt \& pepper, mark, gouge, sliver, scratch, shell, fold, rupture, holes, edge crack and tearing, surface cracks, flatness.

Depending on the line process, defects are acquired at resolutions which can range up to $0.3 \times 0.3 \mathrm{~mm}^{2}$ at $1,200 \mathrm{mpm}$. The detection settings are optimized per product family (requiring specific treatment), so are the multiple classifications, each dedicated to certain ports of the product mix.

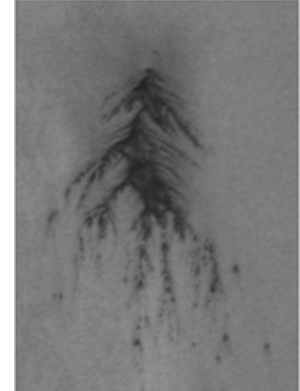

Dirt $(150 \mathrm{~mm} \times$ $300 \mathrm{~mm})$.

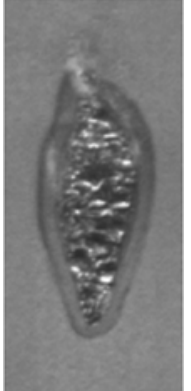

Gouge $(50 \mathrm{~mm} x$ $100 \mathrm{~mm})$.

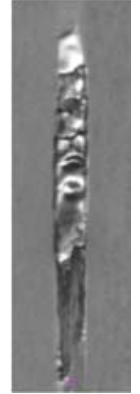

Imprint (35 $\mathrm{mm} x$ $400 \mathrm{~mm})$.

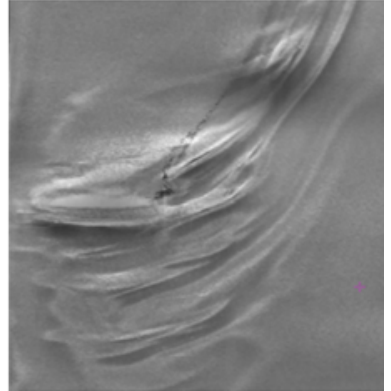

Fold along left edge $(500 \mathrm{~mm} \times 500 \mathrm{~mm})$

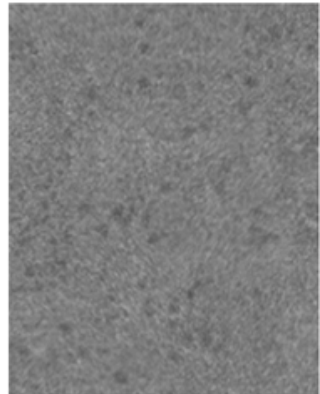

Fine scale (origin: descaling) each spot of scale above is between $1 \& 2 \mathrm{~mm}$

Figure 4. Examples of defects detected by SIAS Next Generation

\subsection{Defects seen by HR and NIR channels}

High Resolution (HR) and Near Infra Red (NIR) channels have been implemented to improve the capability of detecting defects in a more systematic way. The NIR channel is more dedicated to the detection of defects embedded in a highly textured strip surface.

It shall be noted that, by the nature itself of the process, Endless Strip Production lines generate much less individual defects of such type than conventional hot strip mills, which are mostly originated by the transitory periods in the rolling process. Our recent experience shows that only such defect may occur at very start/end of heat sequence, meaning a ratio of at least 7 to 10 in favor of Endless Strip Production compared to conventional hot strip mills in terms of defect occurrence.

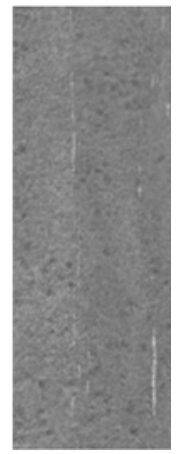

HR - Thin scratches below $1 \mathrm{~mm}$ wide

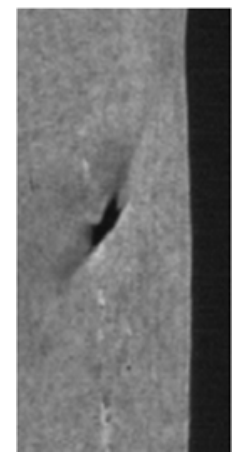

HR - Hole near the edge of the strip (half inch)

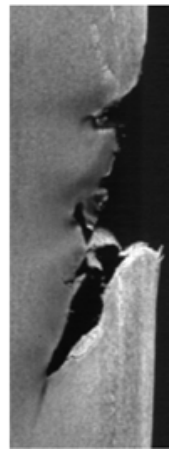

HR - Edge crack $(100 \mathrm{~mm} \times 250 \mathrm{~mm})$
NIR - $30 \mathrm{~mm}$ long shell in infra-red channel

Figure 5. Defects seen by High Resolution (HR) and Near Infra Red (NIR) channels 


\section{TECHNICAL DETAILS ON SIAS}

\subsection{Light source variety and choice of light source type based on the application}

SIAS has been used for a broad variety of hot products such as carbon steel, stainless steel, titanium steel, silicon steel, dual phase products, etc. Some products are very dark, others are very bright. To get a consistent grey level in the picture, SIAS remotely and automatically opens and closes the camera lens aperture depending on the product.

Spot array lighting can also be remotely controlled through TCP/IP from SIAS. SIAS can then optimize the light bar power and lighting profile depending on the type of the product.

For bright products, there is a risk that inspection systems will detect the reflections of the LEDs on the product! To prevent this, SIAS LED beams are anisotropic in order to spread the light in the cross-web direction and not in the down-web direction. This solution is much better than applying a filter to the light bar because it prevents the loss of light that is generated by a filter.

\subsection{Pixel Resolution and video stream pace by the inspection system}

Steel producers demand that smaller and smaller defects be detected, especially very fine scale that indicates the beginning of trouble on the hot strip mills. The pace of the line scan cameras was increased in order to reduce the size of pixels down to $0.3 \times 0.3 \mathrm{~mm}^{2}$. The huge speed output of hot strip mills implies having huge pixel throughput. To get such results, enhancements are at every stage of image acquisition: light bars, camera, frame grabber, and classification process.

Light bar: as a camera line lasts only $20 \mu \mathrm{s}$, the integration time on camera is very short. The latest version of light bars has been recently enhanced with much higher power to match this integration time requirement.

Cameras: of course, cameras have to be very sensitive in order to integrate in such a short time, which is why SIAS uses the up-to-date CMOS technology. Cameras also have to produce a huge amount of data: $80 \mathrm{MHz}$ clock enabling the pace of $0.3 \mathrm{~mm}$ pixel size at 1,200 mpm.

SIAS' Frame grabber - one frame grabber per side - has been updated to grab such a stream based on Xilinx FPGA family 7. SIAS' frame grabber enables a very simple solution: we gather several in one server, which leads to one unique processing machine for majority of a SIAS functions. Algorithms are also able to localize defects in a $500 \mathrm{Mbps}$ stream!

As the amount of pixels per defect increases when the pixel size decreases, the amount of data to process also increases. The latest version of $\mathrm{XLine}^{\circledR}-$ SIAS RT software -provides multi-threaded classification that leads to $100 \mathrm{Mbps}$ to classify.

\subsection{Why Near Infra-Red channel?}

When strip texture is prominent compared to the defects, it is difficult to distinguish the defects from the texture. The latest developments of the infra-red spectrum generated by the strip itself have provided a solution to that problem. Texture is not emitted in IR radiations and thus, defects in near surface or sub-surface are much better revealed as they are not hidden inside the texture.

One dedicated camera is installed in a separate place in order to not be polluted by light from the conventional light channel. This channel provides information about 
surface or subsurface defects such as shells, slivers and imprint scale, but not texture aspects and scale defects.

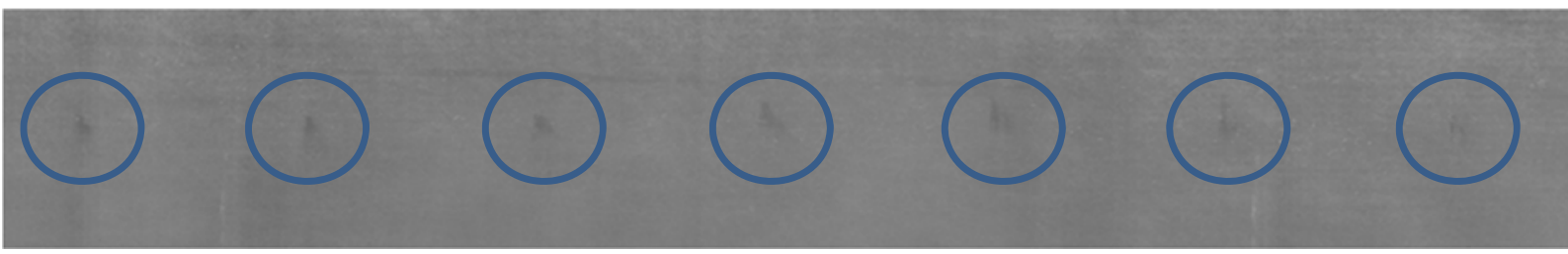

Figure 6. Roll marks with a step of $500 \mathrm{~mm}$ detected in near infra-red channel

The below example show how the NIR channel is used to bring higher contrast for detecting individual defects, which are lost in smaller scale-type defects inside the conventional lighted channel. The post processing defect detection stage is therefore significantly simplified

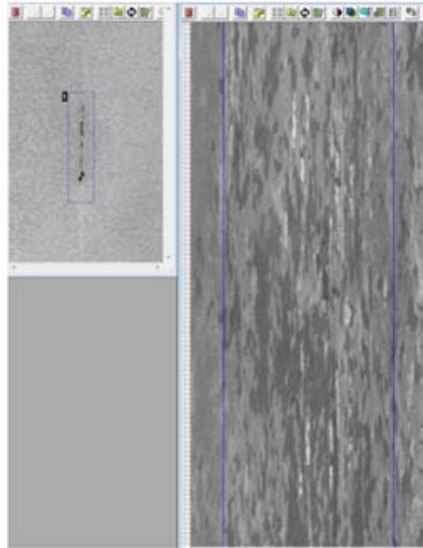

$2 \times 80 \mathrm{~mm}^{2}$ long shell in highly textured background (top left : NIR; right : conventional light)
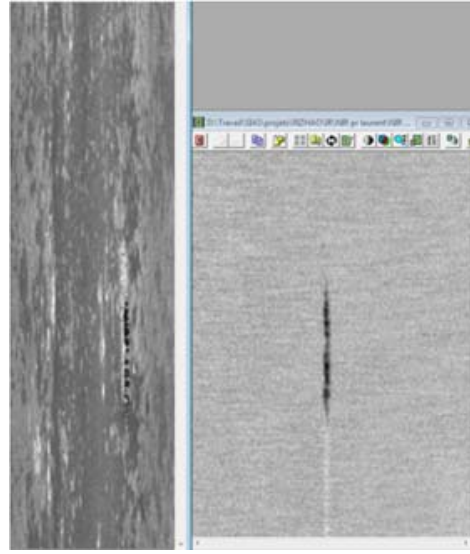

before/after detection processing

$6 \times 100 \mathrm{~mm}$ long shell in highly textured background (left: conventional light; right: NIR)

Figure 7. Comparison between conventional light channel and Near Infra Red (NIR) channel

\section{PERSPECTIVES IN SURFACE INSPECTION AT HOT ROLLING MILLS}

In hot rolling processes (endless and semi-continuous), flatness control remains a key and challenging topic, especially when considering the environment conditions at exit of finishing mill. For flatness measurement, as the first brick of this control chain, Primetals Technologies is addressing such challenges with the use of Hot Planicim $®$ Roll system (HPR). 


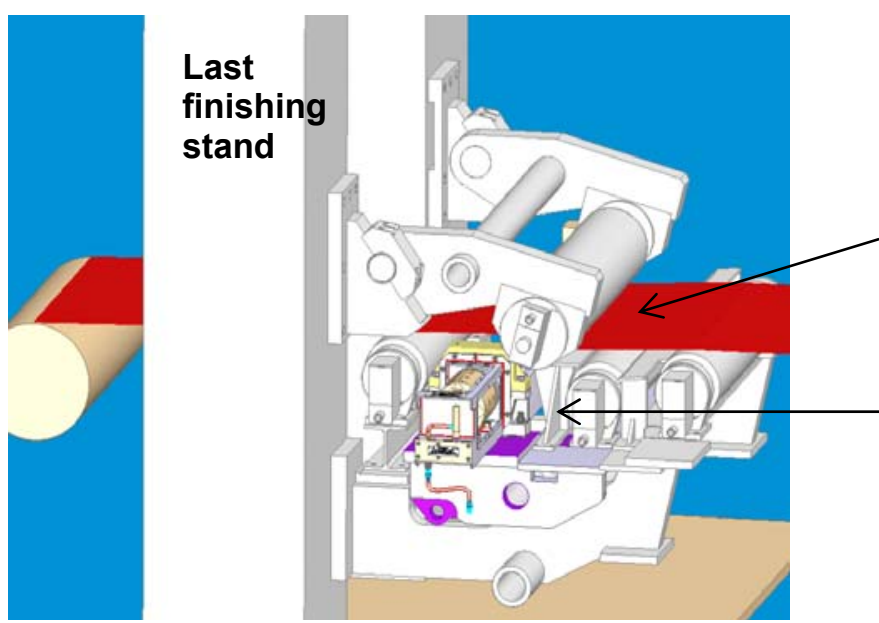

Holddown roll

Figure 8. Hot Planicim Roll system

This mechatronic system is dedicated to the measurement of flatness of hot rolled strip under tension, and located at the exit end of the finishing mill. With a sensitivity of less than $0.5 \mathrm{IU}, \mathrm{HPR}$ provides the relevant alternative information to the usual laser-based flatness gauges which are only able to measure the strip flatness without tension.

The HPR physical measurement principles are based on delivering a continuous image of tension distribution at the exit of the finishing mill, which is converted into a flatness pattern by a further post processing stage. This latest information is therefore used by the operator or the automatic profile and flatness control system to adapt the operating parameters of the last stands.

Jointly with the HPR, the surface inspection system is now evaluated as a valuable source of information for flatness measurement, for instance when addressing flatness variation within limited strip areas (eg extreme edge of strip). The pictures below show typical flatness information from SIAS and HPR from strip under tension. 

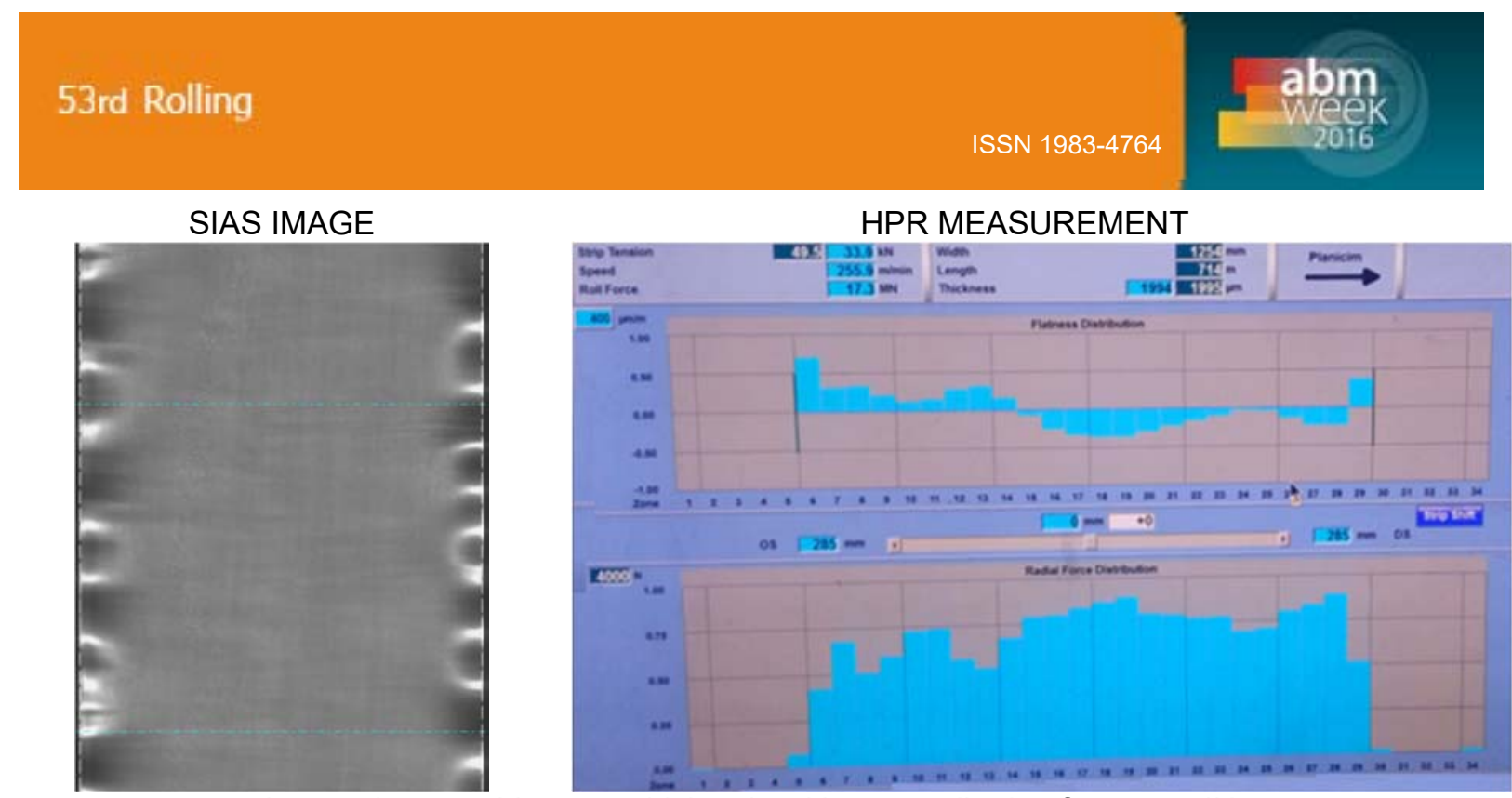

Long edges on this $2 \mathrm{~mm}$ thick strip with an amplitude of 30 to $35 \mathrm{IU}$ while being lightly non symetric as observed on SIAS surface inspection image

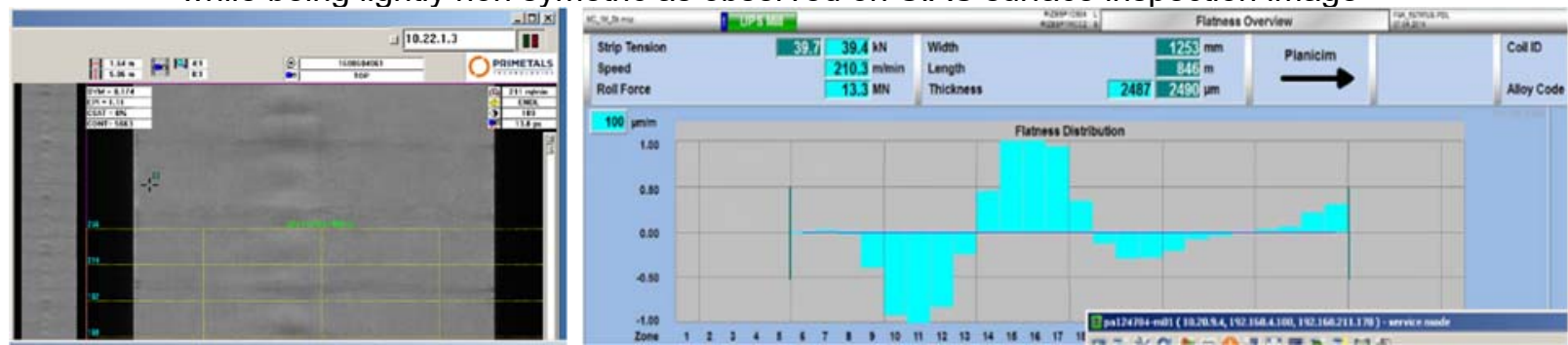

Lightly Off Center buckle of 20 IU on $2.5 \mathrm{~mm}$ hot strip and observed on SIAS surface inspection image

Figure 9. Hot rolled strip typical flatness examples from HPR measurement and SIAS pictures

\section{ONE STEP FORWARD FOR MASTERING GLOBAL QUALITY}

Getting the final required global quality for steel production is the result of successive process steps all along a defined metallurgical route (from the meltshop, through the hot strip mill, pickling line and cold rolling mill down to the processing lines), that individually have a strong influence on the final product quality. Focusing on the final processes is necessary but not sufficient, and this is particularly valid for the high-end automotive market.

This is why, at all critical stages of steel production, Primetals Technologies offers the relevant monitoring systems.

Among required monitoring systems:

- SIAS automatic surface inspection system, with "high-sensitivity" linescan "both sides \& full length" inspection, benefiting from a powerful 3rd generation LED illumination system for high defect \& non defect detection ratio.

- PropertyMon system for non-destructive, real-time and in-line measurement of strip material properties, such as tensile strength and yield strength across the entire length of steel strip.

- Hot Planicim Roll

- Close loop process optimization and product quality tracking various gauges and sensors (roughness, width, thickness, flatness, etc.).

To assist steel makers for quality and process optimization across the entire steel production chain and to valuable and effectively handle the most important available data of different automation and monitoring systems Primetals Technologies has developed a Through Process Quality Control Control system (TPQC). This system is 
a know how rule based system at plant level which can be steadily extended and upgrades by the implementation of know how rules like e.g. metallurgical, numerical and process related rules. Another Primetals Technologies product which can be implemented in the TPQC or can be also installed as a stand-alone version is Primetals Technologies TCOptimizer $囚$. This unique and advanced software is used for just-in-time warnings including a computing intelligence highlighting relevant events without overwhelming users with gigabytes of data. This central expert system collects data and signals from all parts of the production chain, embeds Business Rule Management System (BRMS) based upon simple logical expressions and decision trees to allow non IT-specialists to handle the incoming signals and to manage the generated relevant-only manufacturing events and just-in-time warnings.

\section{CONCLUSION}

Surface inspection remains a cornerstone of quality control. Recent developments in hot rolling have been pursued to bring the earliest relevant information for process optimization, quality tracking, and in the end yield optimization through just-in-time semi-product reallocation.

High resolution inspection has undoubtedly enhanced defect image quality, allowing much better vision and detection of secondary rolled-in scales as well as the very fine, so-called "salt \& pepper" scales. When complemented with Near Infra-Red inspection, the confusion/discrimination between scales and surface texture is eliminated, and the reliable/fast detection of roll marks is achieved. As an additional perspective, surface inspection may also overlap with existing solutions in the field of flatness control.

\section{REFERENCES}

1 Sébastien Maillard, Dr François Mignard, Gaël Imbert, "Processing lines serving highend steel production for automotive products", Estad Conference, Düsseldorf, Germany, June 2015.

2 Myra Pinkham,"Analysis groups" American Metal Market, November-December 2015, pp 18-21

3 Laurie Bonhomme, Benoit Jalard, Stéphane Georges, "Operational feedback from Siemens new process expert system for just-in-time warnings and decision making", 9th International Conference on Zinc and Zinc Alloy Coated Steel Sheet (GALVATECH 2013), Beijing, China, September $2013 .$.

4 SIAS $^{\circledR}$, XLine $^{\circledR}$ and Planicim ${ }^{\circledR}$ are registered trademarks of Primetals Technologies in some countries. 